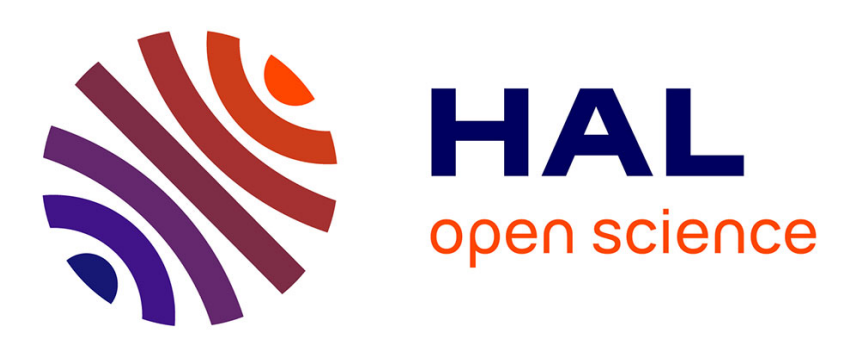

\title{
A detailed comparison of control allocation techniques on a realistic on-ground aircraft benchmark
}

Edouard Sadien, Clément Roos, Abderazik Birouche, Mathieu Carton, Christophe Grimault, Louis Emmanuel Romana, Michel Basset

\section{To cite this version:}

Edouard Sadien, Clément Roos, Abderazik Birouche, Mathieu Carton, Christophe Grimault, et al.. A detailed comparison of control allocation techniques on a realistic on-ground aircraft benchmark. American Control Conference 2019, Jul 2019, PHILADELPHIE, United States. hal-02449260

\author{
HAL Id: hal-02449260 \\ https://hal.science/hal-02449260
}

Submitted on 22 Jan 2020

HAL is a multi-disciplinary open access archive for the deposit and dissemination of scientific research documents, whether they are published or not. The documents may come from teaching and research institutions in France or abroad, or from public or private research centers.
L'archive ouverte pluridisciplinaire $\mathbf{H A L}$, est destinée au dépôt et à la diffusion de documents scientifiques de niveau recherche, publiés ou non, émanant des établissements d'enseignement et de recherche français ou étrangers, des laboratoires publics ou privés. 


\title{
A detailed comparison of control allocation techniques on a realistic on-ground aircraft benchmark
}

\author{
Edouard Sadien ${ }^{\star}$, Clément Roos $^{\dagger}$, Abderazik Birouche ${ }^{\diamond}$, Mathieu Carton ${ }^{\star}$, Christophe Grimault ${ }^{\star}$, \\ Louis Emmanuel Romana ${ }^{\star}$ and Michel Basset ${ }^{\diamond}$
}

\begin{abstract}
To achieve a high performance level during ground operations, the lateral dynamics of an aircraft must be controlled using all available actuators (rudder, nose wheel steering system and brakes), which gives rise to a challenging allocation problem. This provides an excellent benchmark to compare various kinds of control allocation techniques. In this paper, an exhaustive literature review is first presented. The most relevant allocation methods are then applied to an onground aircraft model, which has been previously validated against a high-fidelity Airbus simulator [1]. An extensive evaluation is finally performed based on a set of performance indicators such as the number of iterations, the convergence time, the error and the actuators consumption.
\end{abstract}

\section{INTRODUCTION}

Aircraft control is an important application where actuator redundancy is required, i.e. where the number of actuators must exceed the number of states to be controlled. The need for redundancy is motivated by high performance level requirements, the ability to quickly recover from off-nominal conditions and the tolerance towards actuator failures. This gives rise to control allocation problems, which are often solved by aircraft manufacturers using simple ad hoc techniques. But as the number of control inputs increases, the latter are no longer sufficient, and more systematic allocation methods must be implemented. Several ones are compared in [2] on an airborne aircraft, such as continuoustime and discrete-time versions of direct allocation, linear programming, quadratic programming, as well as variations of the weighted pseudo-inverse approach including a cascaded generalized inverse. But their efficacy is difficult to assess from a general point of view, and it seems preferable to compare them on specific applications [3].

This article moves in that direction and focuses on ground control just after touchdown, which is a particularly demanding phase. Indeed, the aircraft motion is usually controlled manually by the pilot, who must act simultaneously on throttle levers, rudder pedals, handwheels and brake pedals. This is especially demanding in adverse conditions such as contaminated runways and severe crosswinds. So there is a strong motivation to develop enhanced control allocation algorithms able to manage multiple devices with different characteristics. At present, solutions are rare and often only

\footnotetext{
*Airbus Operations S.A.S., Toulouse, France. E-mail: <edouard.sadien, mathieu.carton, christophe.grimault, louis-emmanuel.romana $>@$ airbus.com

†ONERA, The French Aerospace Lab, Toulouse, France. E-mail: clement.roos@onera.fr

•IRIMAS, Université de Haute-Alsace, Mulhouse, France. E-mail: $<$ abderazik.birouche, michel.basset $>@$ uha.fr
}

partial. Several design strategies have been proposed to control the aircraft using the nose-wheel steering (NWS) system only, or less frequently with the rudder as well (see e.g. [4] and [5]). A lot of progress has also been made in the longitudinal energy management and control [6], such as the Brake To Vacate function developed by Airbus [7]. But one of the most challenging on-ground control problems which currently lacks a satisfactory solution - occurs at intermediate speed, where the rudder and the NWS system are less efficient. The main objective is to ensure that the lateral deviation with respect to the runway centerline remains acceptable despite wind, varying runway state and comfort constraints. Achieving good performance during this worstcase scenario makes it necessary to use differential braking in addition to classical control devices (rudder and NWS system), which gives rise to a control allocation problem.

In this context, the main contribution of this paper is to implement the most relevant control allocation techniques available in the literature (see e.g. [8], [3]), to apply them to the aforementioned allocation problem, and to perform a thorough comparison on a realistic worst-case scenario. Using an accurate yet tractable on-ground aircraft model developed in [1], the proposed benchmark takes into account all existing actuators (rudder, NWS system and brakes), including their dynamics and limitations. It also considers changes in the actuators efficiencies due to the variations of normal load and aircraft velocity during the roll-out phase. A set of indicators is introduced to quantify the performance of the on-ground control allocators. It comprises some classical indicators proposed in [9], such as convergence time and number of iterations. Some others are also included based on Airbus expertise in world civil aviation.

The paper is organized as follows. The general control allocation problem is formulated in Section II. The testing framework is thoroughly described in Section III, including the considered scenarios and performance indicators. The various selected methods are briefly described in Section IV. Detailed numerical results are finally presented in Section V.

\section{Control Allocation in A NUTShell}

A two-step strategy is usually applied when an allocation problem arises. First, a high level controller is designed to compute the total control effort that should be applied to fulfill the control objectives. Then, an allocation module is implemented to distribute this effort among the redundant set of actuators. Mathematically, the allocator solves an underdetermined system of equations, often subject to 
additional constraints. It is fed by a vector of virtual inputs $v(t) \in \mathbb{R}^{k}$ (typically a number of forces and moments that equals the number of degrees of freedom to be controlled), and it delivers the true control inputs $u(t) \in \mathbb{R}^{m}$ to be sent to the actuators, where $m>k$. In the literature, effector models are almost always linear in $u$. Thus given $v(t)$, the allocation problem reduces to the computation of $u(t)$ such that:

$$
B u(t)=v(t)
$$

for all $t \geq 0$, where $B \in \mathbb{R}^{k \times m}$ is the control effectiveness matrix of rank $k$. Moreover, actuator rate and position limits are incorporated such that:

$$
u_{\min }^{p} \leq u(t) \leq u_{\max }^{p} \quad, \quad u_{\min }^{r} \leq \dot{u}(t) \leq u_{\max }^{r}
$$

where inequalities apply componentwise. The allocator being embedded in a digital system, rate limits are often converted into effective position limits. The applied limits are then the most restrictive of the position or the converted rate limits:

$$
\begin{aligned}
& \underline{u}(t)=\max \left\{u_{\min }^{p}, u(t-T)+T u_{\min }^{r}\right\} \\
& \bar{u}(t)=\min \left\{u_{\max }^{p}, u(t-T)+T u_{\max }^{r}\right\}
\end{aligned}
$$

where $T$ is the sample time. Therefore, the standard constrained linear control allocation problem is as follows:

$$
B u(t)=v(t) \quad \text { such that } \quad \underline{u}(t) \leq u(t) \leq \bar{u}(t)
$$

However, problem (4) is not convex when sufficient control power is available. In this case, an infinite number of solutions exist and a secondary objective is defined such as minimizing control power. On the contrary, in case of control power deficiency, no exact solution exists and the selected one usually mininizes the $L_{2}$-norm $\|B u-v\|$.

\section{BENCHMARK}

The considered on-ground benchmark is shown in Fig. 1. It is based on the aircraft model described in [1].

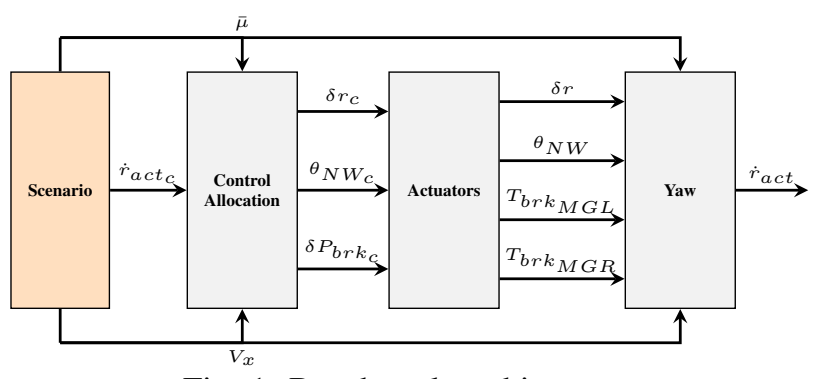

Fig. 1: Benchmark architecture

\section{A. Scenario}

In practice, a dynamic inversion based high level controller computes a virtual input $v=\dot{r}_{a c t_{c}}$ corresponding to the commanded yaw acceleration to be produced by the actuators. This controller is not included in the benchmark, and an $\dot{r}_{a c t_{c}}$ profile recorded during a real landing with difficult operating conditions is used instead. This is indeed a convenient way to push the allocation module to the limits for a better evaluation, and explains why an open-loop benchmark is considered here. In this work, the $\dot{r}_{a c t_{c}}$ issued by the Scenario block corresponds to a landing with $30 \mathrm{kts}$ of crosswind turbulence, as well as $20 \mathrm{kts}$ of lateral gust occurring when the aircraft speed $V_{x}$ is 100,60 and $40 \mathrm{kts}$.

\section{B. Actuators}

The yaw motion of the aircraft is controlled by the braking systems located on the left and right main landing gears (denoted $M G L$ and $M G R$ in the sequel), the NWS system and the rudder, whose models form the Actuators block.

Each braking system is approximated by a first order dynamic model of nominal gain $G_{b r k}$ and time constant $\tau_{b r k}$, with position and rate limits $L_{p b r k}$ and $L_{r b r k}$ :

$\tau_{b r k} \dot{P}_{b r k_{k}}+P_{b r k_{k}}=P_{b r k_{k_{c}}}$ with $\left\{\begin{array}{r}0 \leq P_{b r k_{k}} \leq L_{p b r k} \\ \left|\dot{P}_{b r k_{k}}\right| \leq L_{r b r k}\end{array}\right.$

where $P_{b r k_{k}}$ and $P_{b r k_{k_{c}}}$ are the actual and the commanded braking pressures, and $k \in\{M G L, M G R\}$. Moreover, braking starts only if $P_{b r k_{k}}$ is above a threshold $P_{0}$, so as to overcome the restoring force of springs located between the brake discs and the brake pistons. The torques $T_{b r k_{k}}$ produced by the braking systems are thus obtained as:

$$
T_{b r k_{k}}=\max \left(0, G_{b r k}\left(P_{b r k_{k}}-P_{0}\right)\right)
$$

The NWS (resp. the rudder) actuation system is approximated by a first order dynamic model of unitary gain and time constant $\tau_{N W}$ (resp. $\tau_{\delta r}$ ) with position and rate limits $L_{p N W}$ and $L_{r N W}$ (resp. $L_{p \delta r}$ and $\left.L_{r \delta r}\right)$ :

$$
\begin{gathered}
\tau_{N W} \dot{\theta}_{N W}+\theta_{N W}=\theta_{N W_{c}} \text { with }\left\{\begin{array}{l}
\left|\theta_{N W}\right| \leq L_{p N W} \\
\left|\dot{\theta}_{N W}\right| \leq L_{r N W}
\end{array}\right. \\
\tau_{\delta r} \dot{\delta} r+\delta r=\delta r_{c} \text { with }\left\{\begin{array}{l}
|\delta r| \leq L_{p \delta r} \\
|\dot{\delta} r| \leq L_{r \delta r}
\end{array}\right.
\end{gathered}
$$

where $\theta_{N W}$ and $\theta_{N W_{c}}$ (resp. $\delta_{r}$ and $\delta_{r_{c}}$ ) are the actual and the commanded nose-wheel (resp. rudder) deflections.

\section{Computation of the yaw acceleration}

The Yaw block computes the actual yaw acceleration $\dot{r}_{a c t}$ produced by the actuators. From [1]:

$$
\dot{r}_{a c t}=\dot{r}_{N W}+\dot{r}_{\delta r}+\dot{r}_{b r k_{M G L}}+\dot{r}_{b r k_{M G R}}
$$

The yaw acceleration produced by the rudder is:

$$
\dot{r}_{\delta r}=\frac{q_{d} S c C n_{\delta r} \delta r}{I_{z z}}
$$

where $q_{d}=\frac{1}{2} \rho V_{a}^{2}, S, c, C n_{\delta r}$ and $I_{z z}$ are the dynamic pressure, the reference surface, the mean aerodynamic chord, the yaw stability derivative due to the effect of the rudder deflection $\delta r$ and the inertia around the vertical axis of the aircraft respectively. In addition, $\rho$ is the air density and $V_{a}$ is the Euclidean norm of the aerodynamic velocity.

The yaw acceleration produced by the nose wheel is:

$$
\dot{r}_{N W}=\frac{D_{x N W}}{I_{z z}} \widehat{F_{s y_{N} W}}
$$

where $D_{x N W}$ is the longitudinal distance between the aircraft center of gravity and the nose landing gear, and:

$$
\left.\widehat{F_{s y_{N} W}}=N_{t_{N W}} \text { sat }_{\left[\bar{\mu} \lambda_{s y_{N} W}\right.} \frac{F_{z_{N W}}}{N_{t_{N} W}}\right]\left(K_{y_{N} W} \frac{F_{z_{N W}}}{N_{t_{N W}}} \theta_{N W}\right)
$$


The saturation operator in equation (12) is defined such that $\operatorname{sat}_{[F]}(x)=x$ if $|x|<F$ and $\operatorname{sat}_{[F]}(x)=F \operatorname{sign}(x)$ otherwise. Moreover, $N_{t_{N W}}, \lambda_{s y_{N} W}, F_{z_{N W}}$ and $K_{y_{N W}}$ denote the number of tires, the lateral friction fraction [1], the normal load and the reduced lateral cornering gain respectively. $F_{z_{N W}}$ is obtained by a moment balance at the main landing gears around the lateral axis of the aircraft:

$$
F_{z_{N W}}=\frac{m g D_{x M G}-F_{z a}\left(D_{x M G}-c\left(c_{A}-c_{G}\right)\right)}{D_{x N W}+D_{x M G}}
$$

where $m, g, D_{x M G}$ and $F_{z a}=q_{d} S C z_{0}$ denote the aircraft mass, the standard gravity, the longitudinal distance between the aircraft center of gravity and the main landing gears, and the lift force which depends on the lift stability derivative $C z_{0}$ respectively. In addition, the weight and the aerodynamic effects act at points located along the fuselage axis, whose distances w.r.t. the center of gravity are $-c_{G} . c$ and $-c_{A} . c$ respectively, $c_{G}$ and $c_{A}$ being positive dimensionless coefficients. Finally, $K_{y_{N W}}$ depends on the runway state:

$$
K_{y_{N W}}=\frac{K_{y M A X_{N W}}}{\frac{2}{3}+\frac{1}{3 \bar{\mu}}}
$$

where $K_{Y M A X_{N W}}$ is defined for a dry runway, and the relative friction coefficient $\bar{\mu}$ is considered equal to 1 for dry, 0.74 for wet and 0.29 for snowy runways.

Remark 1: The yaw acceleration produced by the nose wheel rolling resistance is negligible compared to that produced by the lateral slip force and is hence neglected in (11).

The yaw accelerations produced by the left and right braking systems are:

$$
\begin{aligned}
& \dot{r}_{b r k_{M G L}}=-\frac{D_{y M G}}{I_{z z}} F_{s x_{M G L}} \\
& \dot{r}_{b r k_{M G R}}=\frac{D_{y M G}}{I_{z z}} F_{s x_{M G R}}
\end{aligned}
$$

where $D_{y M G}$ is the lateral distance between the aircraft center of gravity and the main landing gears, and:

$$
F_{s x_{k}}=N_{t_{M G}} \text { sat }{ }_{\left[\bar{\mu} \lambda_{s x_{M G}} \frac{F_{z_{M G}}}{N_{t_{M G}}}\right]}\left(\frac{T_{b r k_{k}}}{R_{e}}\right)
$$

where $k \in\{M G L, M G R\} . N_{t_{M G}}, \lambda_{s x_{M G}}, F_{z_{M G}}$ and $R_{e}$ denote the number of tires, the longitudinal friction fraction [1], the normal load and the main landing gear wheel rolling radius respectively. In the same way as before, $F_{z_{M G}}$ is obtained by a moment balance at the nose landing gear around the lateral axis of the aircraft:

$$
F_{z_{M G}}=\frac{m g D_{x N W}-F_{z a}\left(D_{x N W}+c\left(c_{A}-c_{G}\right)\right)}{2\left(D_{x N W}+D_{x M G}\right)}
$$

Remark 2: A complete set of numerical values representative of a commercial aircraft is provided in TABLE I.

\section{Control Allocator}

The control allocator implemented in the Control Allocation block receives the virtual control input $v=\dot{r}_{a c t_{c}}$ and computes the true control inputs $u$ to be sent to the actuators. In order to minimize the effect of differential braking on the longitudinal motion, ganging of the left and right braking

\begin{tabular}{|l|c|c|}
\hline Parameter & Unit & Typical value \\
\hline$m$ & $\mathrm{~kg}$ & $60 \times 10^{3}$ \\
\hline$I_{z z}$ & $\mathrm{~kg} \cdot \mathrm{m}^{2}$ & $3.70 \times 10^{6}$ \\
\hline$S$ & $\mathrm{~m}^{2}$ & 122 \\
\hline$c$ & $\mathrm{~m}$ & 4.2 \\
\hline$c_{G} / c_{A}$ & - & $0.30 / 0.42$ \\
\hline$D_{x N W} / D_{x M G} / D_{y M G}$ & $\mathrm{~m}$ & $11.45 / 1.19 / 3.80$ \\
\hline$C n_{\delta r} / C z_{0}$ & - & $-2.01 / 0.905$ \\
\hline$K_{y M A X_{N W}}$ & $/ \mathrm{rad}$ & 3.56 (wrong value in $[1])$ \\
\hline$R_{e}$ & $\mathrm{~m}$ & 0.50 \\
\hline$\lambda_{s y_{N W}} / \lambda_{s x_{M G}}$ & - & $0.68 / 0.51$ \\
\hline$N_{t_{N W}} / N_{t_{M G}}$ & - & $2 / 2$ \\
\hline$\tau_{b r k} / \tau_{N W} / \tau_{\delta r}$ & $\mathrm{~s}$ & $10^{-3} / 0.1 / 0.2$ \\
\hline$G_{b r k}$ & $\mathrm{Nm} / \mathrm{Pa}$ & $4 \times 10^{-3}$ \\
\hline$L_{p b r k} / P_{0}$ & $\mathrm{~Pa}$ & $175 \times 10^{5} / 15 \times 10^{5}$ \\
\hline$L_{r b r k}$ & $\mathrm{~Pa} / \mathrm{s}$ & $20 \times 10^{5}$ \\
\hline$L_{p N W} / L_{p \delta r}$ & $\mathrm{deg}$ & $74 / 30$ \\
\hline$L_{r N W} / L_{r \delta r}$ & $\mathrm{deg} / \mathrm{s}$ & $20 / 30$ \\
\hline$L_{p b r k_{C A}}$ & $\mathrm{~Pa}$ & $30 \times 10^{5}$ \\
\hline$L_{p N W_{C A}} / L_{p \delta r_{C A}}$ & $\mathrm{deg}$ & $6 / 30$ \\
\hline & & \\
\hline
\end{tabular}

TABLE I: Benchmark values

systems is considered: $\delta P_{b r k_{c}}=P_{b r k_{M G R_{c}}}-P_{b r k_{M G L_{c}}}$ is computed, and $P_{b r k_{M G R_{c}}}=0.5 \delta P_{b r k_{c}}$ and $P_{b r k_{M G L_{c}}}=$ $-0.5 \delta P_{b r k_{c}}$ are then applied in the Actuators block. Thus, the number of outputs of the control allocator reduces to $m=3$ and the matrices of (4) are given by:

$$
\begin{aligned}
& u=\left[\begin{array}{lll}
\theta_{N W_{c}} \delta r_{c} & \delta P_{b r k_{c}}
\end{array}\right]^{T} \\
& v=\dot{r}_{a c t_{c}} \\
& B=\left[\begin{array}{lll}
\frac{\partial \dot{r}_{a c t}}{\partial \theta_{N W}} & \frac{\partial \dot{r}_{a c t}}{\partial \delta r} & \frac{1}{2}\left(\frac{\partial \dot{r}_{a c t}}{\partial P_{b r k_{M G R}}}-\frac{\partial \dot{r}_{a c t}}{\partial P_{b r k_{M G L}}}\right)
\end{array}\right]
\end{aligned}
$$

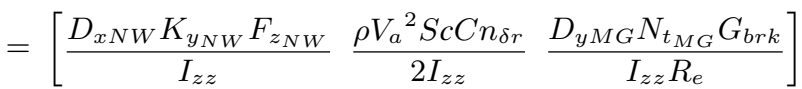

The saturations of the ground reaction forces (12) and (16) are dropped in the computation of the control effectiveness matrix $B$. They are taken into account indirectly by more restrictive actuator position limits:

$u_{\max }^{p}=-u_{\min }^{p}=\left[\begin{array}{lll}L_{p N W_{C A}} & L_{p \delta r_{C A}} & L_{p b r k_{C A}}\end{array}\right]^{T}$

\section{E. Key Performance Indicators}

To be deemed flight worthy, the control allocator should converge in a minimum time (less than the sample time), and in a minimum number of iterations. Most if not all virtual controls should be attained within the capacity of the actuators, therefore minimizing an equivalent yaw acceleration "energy". Lastly, actuator use should be minimized as far as possible. Thus, the following indicators are chosen to assess the performance of the control allocation techniques:

- Mean and maximum number of iterations,

- Mean and average of $10 \%$ maximum convergence time,

- Integral of squared error: $\int\left(\dot{r}_{a c t_{c}}-\dot{r}_{a c t}\right)^{2} d t$,

- Normalized consumption: $\int\left[u_{\max }^{p}{ }^{-1}\right]^{T}|u| d t$.

Remark 3: The first four classical indicators are given per sample time ( $T=40 \mathrm{~ms}$ for the considered benchmark). 


\section{Control Allocation Techniques}

From an exhaustive control allocation literature review including [3], [8], [10], a wide set of methods have been identified. The most appropriate ones are summarized in this section, based on their applicability to the control of an onground aircraft. Indeed, to be considered flight worthy, a control allocation algorithm must reliably produce smoothly varying actuator controls, that do not chatter back and forth from one time step to the next. Furthermore, it should achieve all the virtual control the actuators can produce, or minimize the allocation error in some sense in case of control deficiency [11]. Other factors influencing the choice of some methods over others are determinism, required computing power and certification aspects. Consequently, some techniques are discarded due to their intrinsic chattering likelihood or their potential certification issues. For instance, methods based on model predictive control require that a certain desired trajectory be known during a future horizon, which is incompatible with the existing generalized control law. Moreover, methods based on linear matrix inequalities require a prohibitive computational time, while those using online training neural networks are not compatible with the deterministic criteria of the certification authorities. In the sequel, the time $t$ is omitted unless it is necessary for the understanding of the paper.

\section{A. Unconstrained Methods}

The constraint $\underline{u} \leq u \leq \bar{u}$ is ignored. It means that $B u=v$ has an infinite number of solutions, among which the optimal one in the sense of a certain criterion is selected. Weighted pseudo-inverse [3]: It is the solution of the following minimum 2-norm problem:

$$
\arg \min _{u \in \mathbb{R}^{m}} \frac{1}{2} u^{T} W_{p}^{-1} u \quad \text { subject to } B u=v
$$

where $W_{p} \in \mathbb{R}^{m \times m}$ is a weighting matrix (chosen here as diagonal and composed of the squared position limits of the actuators). The solution is given by:

$$
u=W_{p} B^{T}\left(B W_{p} B^{T}\right)^{-1} v
$$

Frequency-apportioned control allocation [12]: The desired virtual control $v$ is partitioned into low- and highfrequency components using a low-pass filter $L(s)$ :

$$
v_{l}=L(s) v, \quad v_{h}=[1-L(s)] v
$$

A weighted pseudo-inverse is used to allocate both components according to the actuators position and rate limits:

$$
\begin{aligned}
& u_{l}=B_{p}^{\dagger} v_{l}=W_{p} B^{T}\left(B W_{p} B^{T}\right)^{-1} v_{l} \\
& u_{h}=B_{r}^{\dagger} v_{h}=W_{r} B^{T}\left(B W_{r} B^{T}\right)^{-1} v_{h}
\end{aligned}
$$

where $W_{p}$ and $W_{r}$ are weighting matrices (chosen here as diagonal and composed of the squared position and rate limits respectively). The control vector is then given by:

$$
u=\left[B_{p}^{\dagger} L(s)+B_{r}^{\dagger}(1-L(s))\right] v
$$

A key element in this approach is the selection of the lowpass filter time constant. A method is described in [12].
Linear filter [13]: The allocation does not only depend on the current control distribution, but also on the allocation at the previous time step. The following problem is solved:

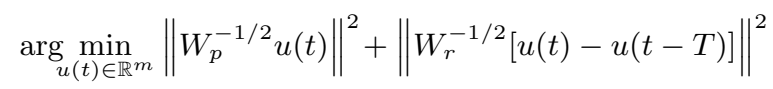

such that $B u=v$, where $W_{p}, W_{r}$ are chosen as above. The solution is:

where:

$$
u(t)=F u(t-T)+G v(t)
$$

$$
\begin{aligned}
& F=(I-G B) W^{-2} W_{r}^{-2} \\
& G=W^{-1}\left(B W^{-1}\right)^{T}\left(\left(B W^{-1}\right)\left(B W^{-1}\right)^{T}\right)^{-1} \\
& W=\left(W_{p}^{-2}+W_{r}^{-2}\right)^{1 / 2}
\end{aligned}
$$

\section{B. Basic Constrained Methods}

Cascaded generalized inverse [14]: It is an iterative heuristics based on the pseudo-inverse method. Position saturated controls are removed from subsequent pseudoinverse solutions until either all control effectors saturate or a solution is found that does not violate actuator constraints.

Daisy chaining [3]: A hierarchy is established between the control effectors, which are separated into different groups. The control inputs are computed assuming that only the first group of effectors is used. If it exceeds the position limits, the overflow is sent to the second group and so on. For the purpose of the benchmark, the first group consists of the classical actuators, i.e. the NWS system and the rudder, and the second one consists of differential braking. Allocation within the first group is done using the pseudoinverse method as described in Section IV-A.

Remark 4: Allocation within the first group can be done with any allocation technique, but the pseudo-inverse method is used here for its low computational power requirement.

\section{Solver-based Methods}

Weighted least squares (LS): It consists of solving:

$$
\arg \min _{\underline{u} \leq u \leq \bar{u}}\|B u-v\|^{2}+\gamma\left\|W_{p}^{-\frac{1}{2}} u\right\|^{2}
$$

where $W_{p} \in \mathbb{R}^{m \times m}$ is a weighting matrix chosen here as in (19) and $\gamma$ is set to a small value, indicating the priority of error minimization over that of control minimization. This can be done using the MATLAB quadprog solver, an active set solver [13], an interior point solver [15] or a fixed-point iteration algorithm [16].

Sequential least squares [13]: It consists of solving the following sequential least squares problem using an active set solver, where $W_{p}$ is chosen here as above:

$$
\arg \min _{u \in \Omega}\left\|W_{p}^{-\frac{1}{2}} u\right\| \quad \text { where } \Omega=\underset{\underline{u} \leq u \leq \bar{u}}{\arg \min }\|B u-v\|
$$

Minimal least squares [13]: A minimal least squares problem (see [17]) formulated in the same way as (28) is solved using a two-stage active set method. $W_{p}$ must be diagonal, which is the case here. However, this implementation does not handle the case of coplanar controls.

Direct allocation [18]: The following problem is solved:

$\max _{\rho \leq 1} \rho$ subject to $B u=\rho v$ and $\underline{u} \leq u \leq \bar{u}$ 
which consists of finding the best approximation of $v$ satisfying the control constraints and being colinear to $v$. An equivalent linear programming problem is derived and solved using the simplex method in [18].

Dynamic allocation [13]: It extends regular quadratic programming control allocation by also penalizing the actuator rates and is formulated as follows:

$$
\begin{gathered}
\arg \min _{u(t) \in \Omega}\left\|W_{p}^{-1 / 2} u(t)\right\|^{2}+\left\|W_{r}^{-1 / 2}[u(t)-u(t-T)]\right\|^{2} \\
\text { where } \Omega=\underset{\underline{u}(t) \leq u(t) \leq \bar{u}(t)}{\arg \min ^{2}}\|B u(t)-v(t)\|
\end{gathered}
$$

where the matrices $W_{p}$ and $W_{r}$ are chosen here as above. Problem (30) is solved using an active set solver in [13].

\section{Iterative Methods}

Nullspace-based pseudo-inverse [19]: The pseudoinverse is used as a primal solution, which is then modified using the nullspace of the control effectiveness matrix. This method handles non-symmetric actuator limits, hence rate limits (see (3)). Moreover, an appropriate solution is given even in the presence of singularity and is guaranteed with a predefined computational burden, which is a non-negligible advantage over linear and quadratic optimization methods.

Fixed point methods [9]: The constrained control allocation problem is formulated into an equivalent fixed point problem. Two algorithms are proposed:

- a sequential method similar to [16], but which adopts a different approach to satisfy the convergence criteria,

- a Newton method, where the consideration of the saturation function leads to a nonsmooth zero finding problem, and which guarantees superlinear convergence.

Dynamical pseudo-inverse [20]: Under the assumption that at least one solution to the allocation problem under saturation exists, two algorithms are proposed which provide a suitable symmetric positive weighting matrix $W$ leading to that solution:

- a linear control allocation algorithm which computes $W$ for the following allocation law linear in $W$ :

$$
u=\left[I+\left(I-B^{T}\left(B B^{T}\right)^{-1} B\right) W\right] B^{T}\left(B B^{T}\right)^{-1} v
$$

- a nonlinear control allocation algorithm which computes $W$ for the following allocation law nonlinear in $W$ :

$$
u=W B^{T}\left(B W B^{T}\right)^{-1} v
$$

$W$ is adjusted only during saturation until values of $u$ are found which satisfy the constraint $\underline{u} \leq u \leq \bar{u}$. However, such an approach does not generally minimize the total control effort, which is often an important requirement.

Karush-Kuhn-Tucker (KKT)-based algorithm [21]: It consists of solving several systems of equations to find all the local optimal solutions, and consequently the global one through a simple comparison between all realistic local minima. This algorithm is independent on the selection of initial conditions, since the considered nonlinear optimization problem is converted into classical eigenvalue problems.

\section{E. Update Law-based Methods}

Dynamic control allocator [22]: It aims at injecting an arbitrary signal in certain input directions, which does not modify the state response or the steady-state output response. This arbitrary signal is the output of a suitable number of integrators, whose state is adjusted online based on certain gains intuitively chosen to promote or penalize the different actuators based on their rate or magnitude saturation levels.

\section{Results And AnAlysis}

The control allocation methods presented in Section IV are now compared on the benchmark described in Section III. The performance indicators mentioned in Section III-E are shown in TABLE II, where N/A means "not applicable". Matlab implementations of all solver-based algorithms, except the Matlab quadprog solver, are available in the $Q C A T$ toolbox [13] and have been slightly adapted for the benchmark. All the other algorithms have been coded from scratch.

All methods except direct allocation make use of a userdefined 3-parameter weighting matrix, which allows the relative minimization of the weighted control vector $W_{p}^{-1 / 2} u$. The frequency-apportioned, the linear filter and the dynamic allocation methods also use an additional weighting matrix $W_{r}$, which penalizes actuator rates. The time constant of the filter $L(s)$ in the frequency-apportioned method depends on the choice of $W_{p}$ and $W_{r}$.

The convergence time should be interpreted with caution, since it was obtained using the MATLAB tic/toc functions, which depend on the CPU consumption of background applications. However, the mean convergence time and mean $10 \%$ maximum convergence times are useful for relative comparison. The former is within the same order of magnitude for all methods except direct allocation, which is almost 10 times slower. A similar comparison was reported in [23].

For the weighted LS problem (27), where the tuning parameter $\gamma$ was set to $10^{-3}$, the active set solver outperforms the MATLAB quadprog solver. Indeed, it uses the solution from the previous sample as an initial guess to hot start the algorithm, which yields faster convergence. The cascaded generalized inverse does not guarantee the full utilization of the actuators' capabilities and may fail to obtain the desired virtual control $v$ if bad choices are made early in the iterations [18]. The number of iterations of the KKTbased algorithm is equal to $3^{m}$, which corresponds to all the combinations of the 3 possible states of each of the $m$ actuators (lower or upper saturated, or not saturated). The fixed-point iteration algorithm [16] converges to the optimal solution as the number of iterations goes to infinity. Here, the maximum number of iterations is limited to 100 and therefore, this method presents the highest allocation error and normalized actuators consumption. Finally, an identity weighting matrix $W=I$ is used by the interior point solver [15] implemented in $Q C A T$. So results could probably be improved by using a diagonal matrix composed of the squared position limits of the actuators, as it is done for several other algorithms. 


\begin{tabular}{|c|c|c|c|c|c|c|}
\hline Methods & $\begin{array}{c}\text { Mean \# of } \\
\text { iterations }\end{array}$ & $\begin{array}{l}\text { Max \# of } \\
\text { iterations }\end{array}$ & $\begin{array}{c}\text { Mean conv. } \\
\text { time }[\mathrm{ms}]\end{array}$ & $\begin{array}{l}\text { Mean } 10 \% \max \\
\text { conv. time }[\mathrm{ms}]\end{array}$ & $\begin{array}{l}\int \text { squared error } \\
{\left[\times 10^{-3} \mathrm{deg} / \mathbf{s}^{3}\right]}\end{array}$ & $\begin{array}{l}\text { Normalized } \\
\text { consumption }\end{array}$ \\
\hline Weighted pseudo-inverse [3] & N/A & N/A & 2.77 & 3.31 & 1.0389 & 81.3319 \\
\hline Frequency-apportioned control allocation [12] & N/A & N/A & 2.82 & 3.36 & 1.2341 & 84.9120 \\
\hline Linear filter [13] & N/A & N/A & 3.27 & 5.10 & 1.6442 & 88.5882 \\
\hline Cascaded generalized inverse [14] & 1.0 & 2 & 2.84 & 3.41 & 1.0389 & 81.3322 \\
\hline Daisy chaining [3] & N/A & N/A & 2.39 & 5.78 & 1.3013 & 75.3898 \\
\hline Weighted LS - quadprog solver & 3.4 & 5 & 7.75 & 10.36 & 1.0562 & 81.6594 \\
\hline Weighted LS - active set solver [13] & 1.0 & 2 & 2.92 & 3.51 & 1.0389 & 81.3228 \\
\hline Weighted LS - interior point solver [15] & 1.7 & 2 & 2.97 & 3.55 & 1.7884 & 108.7635 \\
\hline Weighted LS - fixed-point algorithm [16] & 100 & 100 & 2.97 & 3.55 & 1.8588 & 110.0601 \\
\hline Sequential LS [13] & 2.0 & 3 & 3.08 & 3.68 & 1.0389 & 81.3322 \\
\hline Minimal LS [13] & 2.0 & 3 & 3.00 & 3.63 & 1.0389 & 81.3322 \\
\hline Direct allocation [18] & 1.3 & 2 & 20.42 & 26.33 & 1.6106 & 107.4062 \\
\hline Dynamic allocation [13] & 1.0 & 2 & 3.10 & 3.73 & 1.0430 & 81.3329 \\
\hline Nullspace-based pseudo-inverse [19] & 1.0 & 2 & 3.24 & 4.01 & 1.0389 & 81.3321 \\
\hline Fixed point - sequential method [9] & 28.1 & 47 & 2.97 & 3.60 & 1.0390 & 81.3316 \\
\hline Fixed point - Newton method [9] & 2.0 & 4 & 3.04 & 3.63 & 1.0389 & 81.3315 \\
\hline Linear dynamical pseudo-inverse [20] & N/A & N/A & 2.84 & 3.38 & 1.5504 & 104.1167 \\
\hline Nonlinear dynamical pseudo-inverse [20] & N/A & N/A & 2.86 & 3.40 & 1.5853 & 105.7331 \\
\hline KKT-based algorithm [21] & 27 & 27 & 3.71 & 5.81 & 1.0389 & 81.3321 \\
\hline Dynamic control allocator [22] & N/A & N/A & 3.08 & 3.80 & 1.0359 & 81.8253 \\
\hline
\end{tabular}

TABLE II: Benchmark synthetic results

\section{CONCLUSION}

This paper compares many control allocation techniques on a realistic benchmark, namely the yaw control of an on-ground aircraft. Several performance indicators are computed, such as the number of iterations, the convergence time, the error and the actuators consumption. The most relevant methods in this context are the weighted pseudo-inverse [3], the daisy chaining [3], the linear filter [13] and the dynamic allocator [22] due to their implementation ease and their small convergence time. They require a small computation effort, and they attempt to minimize virtual control error and actuator use. But the weighted pseudo-inverse and the linear filter could lead to disappointing results in case of actuator saturations, which are not taken into account. This issue will be addressed in a future work, as well as robust control allocation and the consideration of actuator dynamics.

\section{ACKNOWLEDGMENT}

This research is supported by Airbus Operations S.A.S., the National Association of Research and Technology (ANRT) and the French Ministry of Higher Education, Research and Innovation under the CIFRE contract 2016/1058.

\section{REFERENCES}

[1] E. Sadien, C. Roos, A. Birouche, C. Grimault, L. E. Romana, J. BoadaBauxell, and M. Basset, "Control design oriented modeling of an onground aircraft," in Proceedings of the ECC, 2018, pp. 2757 - 2762.

[2] A. Page and M. Steinberg, "A closed-loop comparison of control allocation methods," in Proceedings of the AIAA GNC, 2000.

[3] M. Oppenheimer, D. Doman, and M. Bolender, "Chapter 8: Control allocation," in The Control Handbook, Control System Applications (Second Edition), W. S. Levine, Ed. CRC Press, 2010, pp. $1-24$.

[4] C. Roos, J.-M. Biannic, S. Tarbouriech, C. Prieur, and M. Jeanneau, "On-ground aircraft control design using a parameter-varying antiwindup approach," AST, vol. 14, no. 7, pp. $459-471,2010$.

[5] D. Lemay, Y. Chamaillard, M. Basset, and J. Garcia, "Gain-scheduled yaw control for aircraft ground taxiing," in Proceedings of the 18th IFAC World Congress, 2011, pp. 12970 - 12975.

[6] J. Duprez, F. Mora-Camino, and F. Villaumé, "Control of the aircrafton-ground lateral motion during low speed roll and manoeuvers," in Proceedings of the Aerospace Conference, 2004, pp. 2656 - 2666.
[7] F. Villaumé and T. Lagaillarde, "Runway overrun prevention system," Airbus, Tech. Rep. 55, 2015, www.airbus.com/content/dam/corporatetopics/publications/fast/Airbus-FAST55.pdf.

[8] T. Johansen and T. Fossen, "Control allocation - A survey," Automatica, vol. 49, no. 5, pp. 1087 - 1103, 2013.

[9] A. Naskar, S. Patra, and S. Sen, "New control allocation algorithms in fixed point framework for overactuated systems with actuator saturation," IJC, vol. 90, no. 2, pp. 348 - 356, 2017.

[10] T. I. Fossen, T. A. Johansen, and T. Perez, "Chapter 7: A survey of control allocation methods for underwater vehicles," in Underwater Vehicles, A. V. Inzartsev, Ed. InTech, 2008, pp. $109-128$.

[11] D. Enns, "Control allocation approaches," in Proceedings of the AIAA GNC, 1998.

[12] J. Davidson, F. Lallman, and T. Bundick, "Integrated reconfigurable control allocation," in Proceedings of the AIAA GNC, 2001.

[13] O. Härkegård, "Backstepping and control allocation with applications to flight control," Ph.D. dissertation, Linköping University, 2003, http://research.harkegard.se/qcat.

[14] K. Bordignon, "Constrained control allocation for systems with redundant control effectors," Ph.D. dissertation, Virginia Polytechnic Institute and State University, 1996.

[15] J. Petersen and M. Bodson, "Constrained quadratic programming techniques for control allocation," IEEE TCST, vol. 14, no. 1, pp. 91-98, 2006.

[16] J. Burken, P. Lu, Z. Wu, and C. Bahm, "Two reconfigurable flightcontrol design methods: Robust servomechanism and control allocation," JGCD, vol. 24, no. 3, pp. $482-493,2001$.

[17] P. Lötstedt, "Solving the minimal least squares problem subject to bounds on the variables," BIT Numerical Mathematics, vol. 24, pp. $206-224,1984$.

[18] M. Bodson, "Evaluation of optimization methods for control allocation," JGCD, vol. 25, no. 4, pp. $703-711,2002$.

[19] D. Buzorgnia and A. Khaki-Sedigh, "Constrained dynamic control allocation in the presence of singularity and infeasible solutions," ArXiv e-prints, 2016.

[20] J. Bošković and R. Mehra, "Control allocation in overactuated aircraft under position and rate limiting," in Proceedings of the ACC, 2002, pp. $791-796$.

[21] Y. Chen and J. Wang, "Fast and global optimal energy-efficient control allocation with applications to over-actuated electric ground vehicles," IEEE TCST, vol. 20, no. 5, pp. 1202 - 1211, 2012.

[22] L. Zaccarian, "Dynamic allocation for input redundant control systems," Automatica, vol. 45, no. 6, pp. 1431 - 1438, 2009.

[23] Y. Ikeda and M. Hood, "An application of L1 optimization to control allocation," in Proceedings of the AIAA GNC, 2000. 